\title{
MANEJO DE PARASITOSES EM SISTEMA ORGÂNICO DE PRODUÇÃO DE LEITE
}

\author{
L.R. de Almeida*
}

Fiocruz, Av. Brasil, 4365, CEP 21040-360, Manguinhos, RJ, Brasil. E-mail: rodrigues_lu@yahoo.fr

\author{
RESUMO
}

\begin{abstract}
Os sistemas agropecuários de produção orgânica respondem às necessidades do mercado e às crescentes preocupações dos consumidores, relacionadas à qualidade dos alimentos, ao respeito ao meio ambiente e ao bem-estar dos animais. Em tais sistemas de produção animal, o emprego de produtos químicos de síntese é severamente limitado. Assim, a conversão de um sistema de produção animal convencional em sistema de produção orgânica é acompanhada de alterações relacionadas à intensidade e à diversidade de infestação/infecção dos animais por ecto e endoparasitos, tornando indispensável o emprego de medidas profiláticas. O presente trabalho tem como objetivo expor as principais questões relacionadas ao manejo de parasitoses em sistemas orgânicos de produção de leite, assim como suas perspectivas de controle.
\end{abstract}

PALAVRAS-CHAVE: Produção orgânica de leite, saúde animal, parasitoses.

\section{ABSTRACT}

PARASITESMANAGEMENTINDAIRY ORGANICSYSTEM. Organic farming systems should meet the needs of markets as well as satisfy the emerging concerns of consumers about food quality, environmental issues and animal welfare. In such production systems, the use of synthetic chemical products is severely restricted. The conversion of conventional animal production systems into organic ones should encompass changes in the diversity and intensity of infection by parasites. Moreover, the use of prophylactic practices becomes imperative. The aim of this article is to set forth the main questions associated to parasite management in organic dairy production systems as well as their control perspectives.

KEY WORDS: Dairy organic farming, animal healthy, parasites.

De acordo com a Regulamentação da Produção Animal Orgânica (BRASIL, 2003; REPAB, 2007), na produção animal orgânica devem ser empregadas as terapêuticas homeopática, fitoterápica e a acupuntura, sendo restrito o uso de produtos químico de síntese. Tal exclusão do emprego de drogas sintéticas determina a necessidade de manejo voltado fundamentalmente à prevenção das enfermidades. Neste tipo de sistema, o manejo da criação animal tem como princípios o respeito ao bem-estar animal e sua qualidade de vida, sendo assim necessário dispor de instalações funcionais e confortáveis, com alto nível higiênico, em todo o processo criatório. Como nos sistemas convencionais, são obrigatórias as vacinas estabelecidas por lei e recomendadas as vacinações para as doenças mais comuns em cada região. Na criação orgânica, éimprescindível o uso de raças adaptadas, mais rústicas, capazes de produzir satisfatoriamente em condições naturais de criação, sem o uso preventivo de antibióticos, promotores de crescimento e implantes hormonais. Devem-se buscar melhores resultados na produção, sem pôr em risco a qualidade de vida dos animais, devido ao estresse causado por metas de altos índices de produção. Assim, a concentração de animais deve ser reduzida, em comparação aos sistemas convencionais. Evidentemente, tais condições favoráveis atuarão de forma sinérgica ao funcionamento do sistema imunológico dos animais e, consequentemente, à saúde do rebanho. No contexto do modelo convencional, a intensificação da produção animal propicia o desenvolvimento de doenças de naturezas diversas: infecciosas, metabólicas, parasitarias, imunológicas (RAGOT, 2000), comportamentais e etc., exigindo emprego de medicação sistemática

\section{Definição do estado saúde dos animais em sistema produção orgânica}

O estado de saúde dos animais de produção pode ser definido como um estado de bem-estar e

*Pesquisadora visitante IOC/Fiocruz. 
de equilíbrio entre um organismo e seu meio, sendo, a doença, resultado da inadaptação do animal ao meio. Assim, saúde, em produção animal, significa considerar as necessidades físicas, fisiológicas e psíquicas (comportamentais) do animal. Devem ser respeitadas as condições satisfatórias de vida, permitindo-lhes a otimização do seu potencial genético em condições ecomicamente rentáveis e desprovidas de qualquer risco ou inconveniente para o consumidor. Deve-se aceitar um nível razoável de produção (RAGOT, 2000).

Principal entrave associado à conversão de um sistema convencional de produção animal para sistema orgânico, em relação aos aspectos sanitários

Evidentemente, a conversão de sistema de produção animal convencional em sistema de produção orgânica é acompanhada do risco emergente de infecção/infestação dos animais por endoparasitos e ectoparasitas, até então controlados por produtos químicos de síntese e restritos quando empregado sistema de confinamento. Assim, a utilização de animais mestiços terá grande importância, uma vez que os bovinos com maior grau de sangue indiano são mais resistentes a endo e ectoparasitas. Ainda que uma produção menos intensa seja aceita na agropecuária orgânica, as soluções economicamente viáveis para o sistema devem basear-se, como nos sistemas convencionais, em medidas que visem à prevenção das parasitoses (HOSTE; DoRCHIES, 2000; WALLER; THAMSBORG, 2004).

\section{Possibilidades de controle de parasitos em sistemas orgânicos de produção}

Como nos sistemas de produção convencional, a ocorrência das doenças parasitárias está na dependência, também, de fatores como a presença de hospedeiros intermediários e vetores, estado decontaminação dos piquetes e instalações e pelo número de larvas e ovos presentes nesse ambiente. Assim, a higiene dos estábulos, bezerreiros e bebedouros será sempre um fator fundamental para a prevenção das parasitoses. Para o controle, a associação de práticas como manejo de pastagens visando à redução da infestação/infecção por parasitos; utilização de raças geneticamente resistentes às parasitoses; nutrição adequada; emprego de práticas decontrole biológico através da utilização defungos parasitófagos (LARSEN, 1999) e o uso de medicamentos homeopáticos e fitoterápicos representam uma perspectiva em sistemas de produção orgânica. A limitação da lotação das parcelas (menos de 15 a 30\% de animais por área em relação aos sistemas convencionais) (Fig. 2) regulamentada pelas normas de produção orgânica atua como fator amenizante do risco de infestação/ infecção por parasitos, favorecendo o controle do parasitismo em sistemas orgânicos (RAGOT, 2000).

\section{Homeopatia}

A idéia central da homeopatia está baseada no princípio da similitude: os efeitos de substâncias ultradiluídas são estudados em indivíduos sadios e descritos nas Matérias Médicas Homeopáticas. Quando um paciente enfermo apresenta sintomas semelhantes aos produzidos pela substância no indivíduo sadio, esta substância (medicamento homeopático) é empregada em busca da cura. Tal fenômeno constitui o princípio fundamental da homeopatia: "Cura pelo Semelhante" (HAHNEMANN, 1835). Para a homeopatia, doença é a manifestação de sintomas resultante da tentativa do organismo de restabelecer seu equilíbrio interno, o qual foi " perturbado" por estímulosindesejáveis (HAHNEMANN, 1835). Assim, podemos entender como fatores estressantes presentes nos sistemas de produção animal: alta taxa de lotação, confinamento, impossibilidade de expressar os padrões normais de comportamento da espécie, entre outros.

A prescrição homeopática baseia-se na origem da enfermidade(dependendo da origem da enfermidade, em alguns casos, pode ser empregado o mesmo medicamento para todo o rebanho), no comportamento do animal enfermo (se este quando enfermo se apresenta mais agressivo, se apresenta tendência a se isolar do grupo, se deseja ser tocado ou não e etc.) e em características biotipológicas, as quais possibilitam conhecer as predisposições mórbidas das raças e dos animais individualmente, ou seja, a quais doenças um animal de determinado biótipo é predisposto, o que possibilita a adoção de medidas profiláticas e orienta o tratamento (adaptado de CARILLO JUNIOR, 2000). A homeopatia fornece possibilidades de cura infinitamente superiores, quando comparada à alopatia, a qual atua, principalmente, através da supressão de sintomas, não possibilitando a cura. Vale ressaltar que o custo do medicamentohomeopáticoébaixo, constituindo outra vantagem para o produtor. Em relação à aplicação dos medicamentos homeopáticos, estes podem ser adicionados ao alimento no cocho, à mistura mineral ouaplicados individualmente aos animais, via mucosa oral ou vaginal, através de borrifador (Fig. 1) (PINTO; AlmeIDA, 2002; CAstilhos et al., 2003)

Controle das parasitoses em sistemas orgânicos de produção animal: aplicabilidade da homeopatia e da fitoterapia

A homeopatia e a fitoterapia, embora fundamentalmente distintas, apresentam inúmeras aplicações em medicina veterinária, principalmenteem sistemas de produção orgânica, nos quais estas constituem 
as terapêuticas de primeira escolha. Entretanto, a fitoterapia, assim como a medicina tradicional, considera os micro-organismos e os parasitos como agressores, nãoconsiderando a receptividade própria do paciente (LABRE, 2001). O desenvolvimento de uma parasitose implica que o indivíduo infestado/ infectado apresente condições para a sobrevivência e reprodução do parasita (CARILLO, 2000). O medicamento homeopático é capaz de atuar estimulando a reação imunológica do hospedeiro parasitado, assim como tratar os distúrbios de origem parasitária, pode atuar também modificando a receptividade dos animais ao parasitismo. Porém, nenhum medicamento homeopático é conhecido por sua ação direta contra os parasitos (LABRE, 2001). Assim, diferentemente da homeopatia, os medicamentos fitoterápicos conhecidos por terem ação contra parasitos atuam diretamente contra estes (ATHANASIADOU et al., 2000; Olavez et al., 2001; SANCHÉz et al., 2001). Entre as vantagens do emprego da fitoterapia, destacam-se os aspectos econômicos e a possibilidade da administração do princípio ativo sob a forma de pastagem (Villalba et al., 2010). Porém, devem ser aplicados os mesmos cuidados recomendados para os produtos químicos de síntese, a fim de evitar o aparecimento do fenômeno de resistência.

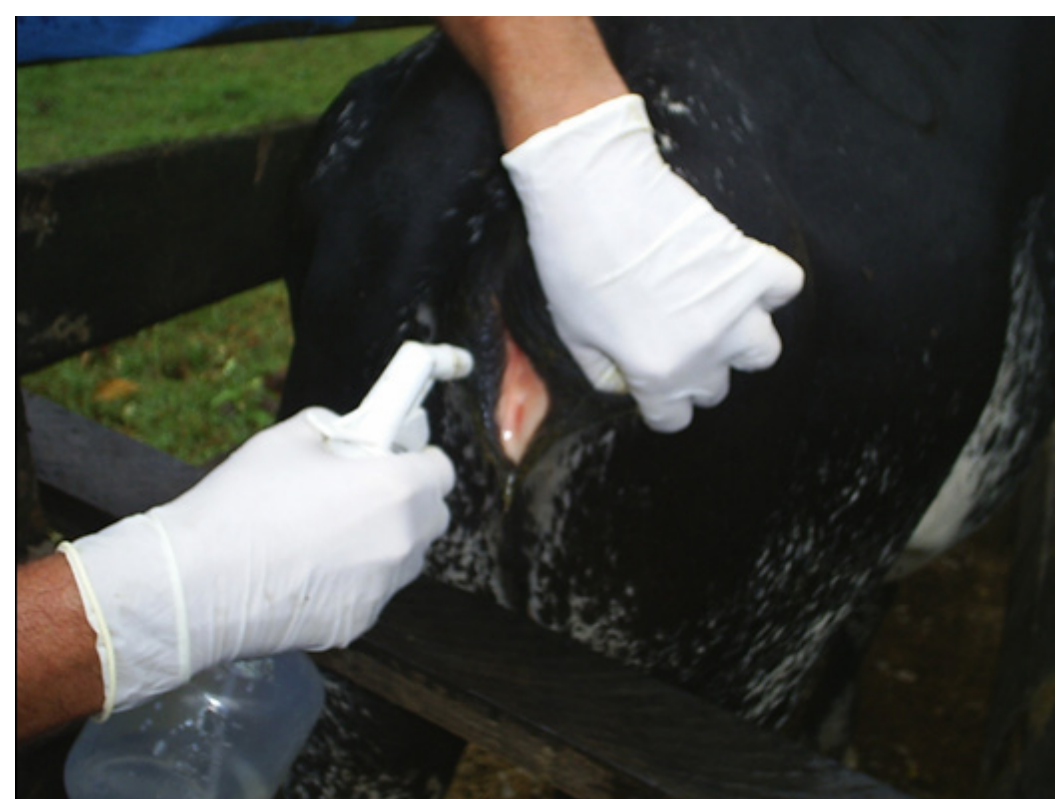

Fig. 1 - Aplicação do medicamento homeopático, via mucosa vaginal, através de borrifador plastico (PINTO; AlmEIDA, 2002; CAstiLhos et al., 2003).

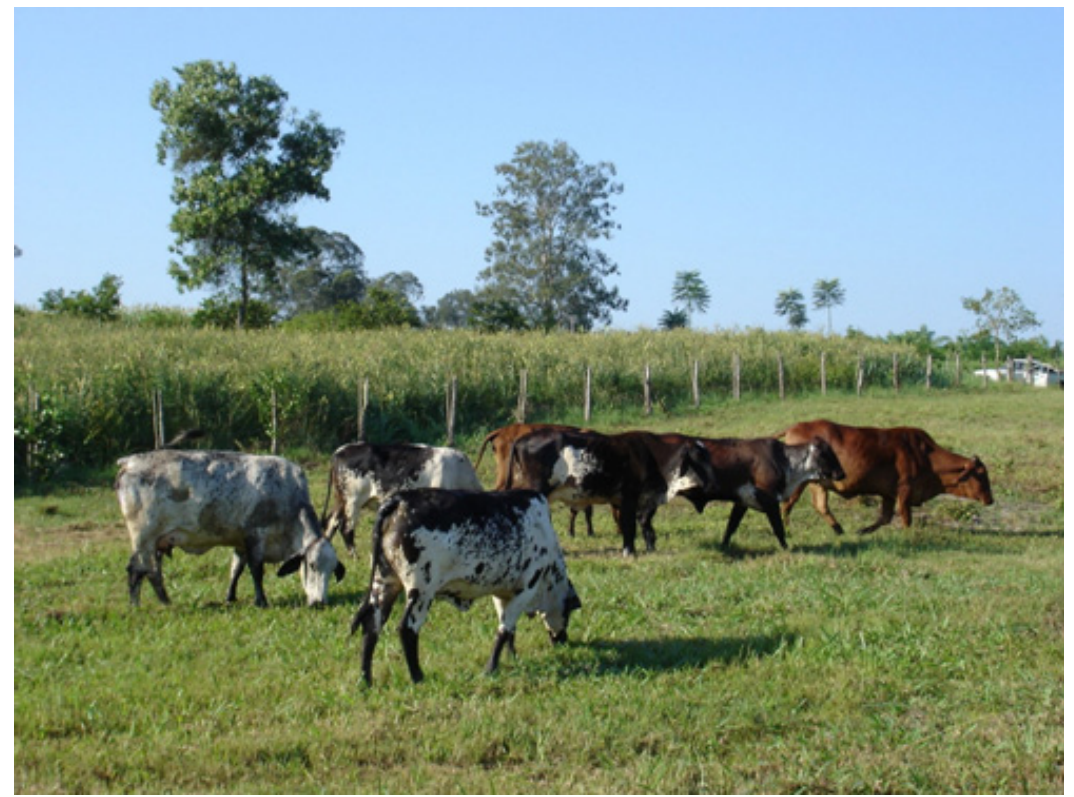

Fig. 2 - Rebanho bovino formado por animais mestiços sob manejo orgânico. Limitação da taxa de lotação. Projeto Fazendinha, destinado à produção orgânica de alimentos (Embrapa-CNPAB/UFRRJ / PESAGRO-RJ). 


\section{Possibilidades de controle da verminose dos animais em sistemas orgânicos}

O controle das helmintoses é de interesse prioritário em sistemas de produção orgânica. A ênfase é dada à prevenção não química (CABARET et al., 2002a; CABARET et al., 2002b), sendo a utilização de drogas anti-helmínticas sintéticas estritamente limitada em tais sistemas de produção.

Cabe ressaltar que a prevenção será sempre o aspecto mais importante em relação ao tratamento. Sendo assim, além da utilização de raças resistentes às verminosese bem adaptadas ao ambiente, constituem práticas fundamentais: remover continuamente fezes nas instalações, evitar acúmulo de umidade, evitar que os animais jovens entrem em contato com acúmulo de fezes dos animais adultos, uma vez que os adultos resistem melhor às infecções por vermes, porém, liberam ovos de helmintos no ambiente, os quais irão infectar os animais jovens que são mais susceptíveis e provavelmente adoecerão (AlmEIDA, 2004). Em relação às possibilidades de tratamento homeopático, o uso do medicamento homeopático Sulphur (enxofre dinamizado) constitui uma possibilidade de tratar a susceptibilidade dos animais ao parasitismo, podendo ser empregado também na prevenção da verminose. Outros medicamentos homeopáticos como Cina e Spigellia também devem ser considerados. Entre os vermífugos fitoterápicos, existem relatos sobre o uso de dentes de alho, sementes de abóbora, folhas de Erva-de-Santa-Maria (Chenopodium ambrosioides) (SANCHÉZ etal., 2001), além de plantas que contêm taninos condensados (Hedysarum coronarium, Lotus pedunculatus) (ATHANASIADOU et al., 2000). Entretanto, faltam ainda informações técnicas precisas relacionadas às quantidades, frequência de administração, entre outros.

Manejo das pastagens visando ao controle da verminose de ruminantes

A limitação do tratamento anti-hemíntico em sistemas de produção orgânica "exige" que os animais pastem o máximo possível em pastagens livres de parasitos. Assim, os métodos de manejo racional de pastagens baseiam-se no seguinte princípio: reduzir a fonte de parasitos em contato com animais sensíveis (Hoste; CHARTIER, 1997). Entre as possibilidades estão: a utilização de áreas anteriormente empregadas em cultivo de cereais para o pastejo dos animais; a alternância anual de ovinos e bovinos nas pastagens; pastejo alternado entre equinos e pequenos ruminantes; pastejo alternado entre bovinos adultos e pequenos ruminantes; pastejo alternado ou misto entre animais da mesma espécie, porém, de diferentes idades, e pastejo rotacionado (Hoste; Dorchies, 2000). A rotação de pastagens é uma prática zootécnica, normalmente empregada para garantir a qualidade nutricional das forrageiras, podendo ser também utilizada com interesse de controle do parasitismo, tendo como princípio a descontaminação através do descanso da pastagem. A prática determina o repouso da pastagem durante certo tempo, determinado de acordo com a ação das condições climáticas da região sobre as fases de vida livre dos parasitos.

Embora procedimentos simples, baseados no conhecimento epidemiológico, sejam capazes de fornecer significantes benefícios ao controle das helmintoses, estes têm sido pobremente adotados (WALLER, 1997).

\section{Tratamento com vermífugos químicos conven- cionais}

Caso, em algum momento, seja julgado indispensável o tratamento convencional (com produtos químicos de síntese), após diagnóstico do(s) verme(s) em questão, deve-se preferir os anti-helmínticos de administração oral, de restrito espectro, como, por exemplo, os tem que como princípio ativo o closantel, específico para infecções por Haemonchus sp., o qual é, normalmente, o verme que causa maiores prejuízos para a saúde dos animais de produção (bovino, caprino e ovino). Em seguida, deve-se retirar temporariamente o animal tratado do sistema, até que o medicamento sintético seja completamente eliminado. Cabe ressaltar que, em produção orgânica, tal tratamento somente poderá ser efetuado após diagnóstico da parasitose, nunca de forma preventiva e sempre sob supervisão do médico veterinário.

\section{Possibilidades de controle da infestação por carrapatos}

Para controle das infestações por carrapatos, devem ser adotadas práticas de manejo visando reduzir o nível de infestação do ambiente (pastagens). Dentre as medidas mais eficazes, destacam-se o rodízio de pastagens com descanso de pelo menos 30 dias por piquete. A utilização de raças resistentes ao carrapato e bem adaptadas também contribui para o controle, sendo os bovinos com maior grau de sangue indiano mais resistentes a ectoparasitas. Para tratamento e prevenção das infestações o uso do medicamento homeopático Sulphur (enxofre dinamizado) constitui uma possibilidade de tratar a susceptibilidade dos animais ao parasitismo (ALMEIDA et al., 2007). Entre as possibilidades de controlar o carrapato através do uso de plantas, existem relatos sobre o uso de pulverizações contento fumo, timbó, neem (Olavez et al., 2001), além de piretroides naturais. Entretanto, faltam ainda informações técnicas precisas relaciona- 
das às quantidades, frequência de administração etc. O controle dos carrapatos através da catação manual também deve ser considerado, sendo aplicável ao gado de leite, por ocasião da ordenha, possibilitando a redução da infestação de carrapatos na propriedade a longo prazo.

Possibilidades de controle da infestação por berne (Dermatobia hominis)

Para controle da infestação por bernes, algumas práticas de manejo são muito importantes, como: reduzir a presença de moscas domésticas nos estábulos através do manejo correto de composteiras e do esterco nas proximidades das instalações; buscar selecionar, entre os animais do rebanho, aqueles que se apresentam resistentes à infestação por bernes, sem deixar de levar em consideração, é claro, o potencial produtivo destes animais. Em relação às possibilidades de tratamento homeopático, mais uma vez, o uso do medicamento homeopático Sulphur (enxofre dinamizado) constitui uma possibilidade de tratar a susceptibilidade dos animais ao parasitismo, podendo ser empregado também na prevenção das infestações (AlmEIDA et al., 2007). Pode ser empregado também medicamento homeopático preparado a partir de larvas de bernes (nosódio), o qual atuará estimulando a imunidade específica do organismo contra as larvas. Podem ser empregadas, também, práticas fitoterápicas, através de pulverizações contendo piretroides naturais (suco de piretro), florde-enxofre, citronela e capim-limão Porém, estudos mais precisos devem ser ainda dedicados à avaliação criteriosa dos efeitos de tais substâncias, assim como à determinação das doses e frequência de aplicação.

Manejo nutricional e susceptibilidade dos animais à verminose

"Alimente seus animais e eles se ocuparão de seus parasitos". Existe uma competição denutrientes entre a resposta imune durante infecção por endoparasitos e as exigências nutricionais do animal para a manutenção de outras funções corporais (DORCHIES, 2000). As perdas nutricionais ocasionadas pelas verminoses acometem principalmente o metabolismo das proteínas, em comparação ao metabolismo energético dos animais. É evidente que a subnutrição favorece o parasitismo (CoOP; HolmEs, 1996). Uma nutrição adequada em quantidade e qualidade permite compensar, ao menos parcialmente, a má-absorção dos nutrientes provocada pela presença dos parasitas no tubo digestivo dos animais parasitados. Uma alimentação equilibrada determina, também, melhor resistência do hospedeiro às infecções, ocasionando redução da instalação e da fertilidade dos parasitos. Alguns autores observaram redução da infestação parasitária e melhora significativa da performance dos animais com a adição de certas leguminosas ricas em taninos condensados (em particular Lotus pedunculatus) na alimentação de pequenos ruminantes (CoOP; KYriazaKis, 1999).

Medidas sanitárias profiláticas gerais de manejo, indispensáveis em uma unidade de produção de leite orgânico

Entre as práticas básicas de manejo, as quais visem à profilaxia das enfermidades que acometem mais comumenteorebanholeiteiro, estão: cuidadoscomanimal recém-nascido (desinfecção do umbigo e ingestão do colostro); higiene no processo de ordenha (antes, durante e após), além de evitar que as vacas deitem logo após a ordenha, quando o esfíncter da teta ainda se encontrará aberto; higiene das instalações, sendo imprescindível a remoção contínua das fezes nos currais; evitar que os animais jovens entrem em contato com as fezes dos adultos, devido ao risco de infecção por vermes, além de evitar a associação de espécies na mesmainstalação, devidoàs diferençasentreasespécies em relação à susceptibilidade a doenças.

A associação de animais de diferentes espécies na mesma instalação ("manejo Arca de Noé"), por vezes empregada em sistemas de produção orgânica, deve ser considerada com cautela sob os aspectos sanitários devido às diferenças entre as espécies em relação à susceptibilidade a determinados agentes patogênicos. Por exemplo, em relação à associação de bovinos e suínos, a presença do nematódeo Ascaris suum, o qualé normalmente encontrado no intestino de suínos sem comprometer a saúde destes, pode determinar doença pulmonar grave em bovinos (BloOD; Radostits, 1991).

\section{CONCLUSÃO}

No manejo sanitário dos animais em sistema orgânico de produção de leite, observa-se que não existe prática ideal de controle das parasitoses, entretanto, os métodos disponíveis, quando associados e bem empregados, podem resultar na redução ou exclusão do uso de antiparasitários químicos de síntesee, consequentemente, na diminuição da poluição ambiental, diminuição de resíduos nos produtos de origem animal e na redução da dependência do produtor em relação à aquisição de insumos externos à propriedade.

\section{REFERÊNCIAS}

ALMEIDA, L.R.; FONSECA, A.H.; OLIVEIRA, C.J.F. Perspectivas de controle de nematódeos gastrointesti- 
nais de ruminantes. Revista Brasileira de Medicina Veterinária, v.26, p.74-78, 2004.

ALMEIDA, L.R.; SILVA, F.S.; FONSECA, A.H.; SOARES, J.P.G.; LUCKE, I. Tratamento homeopático da infestação por Dermatobia homins (Linnaeus Jr, 1781) (Díptera: Cuterebridae) em bovinos sob manejo orgânico. Seropédica, RJ: Embrapa Agrobiologia, 2007. 4p. (Embrapa Agrobiologia. Comunicado Técnico, 104).

ATHANASIADOU, S.; KYRIAZAKIS, I.; JACKSON, F.; COOP, R.L. Consequences of long-term feeding with condensed tannis on sheep parasitised with Trichostrongylus colubriformis. International Journal for Parasitology, v.30, p.1025-1033, 2000.

BLOOD, D.C.; RADOSTITS, O.M. Clínica veterinária. 7.ed. Rio de Janeiro: Guanabara Koogan, 1991. 1263p.

BRASIL. Lei n ${ }^{\circ}$ 10,831 de 23 de dezembro 2003. Dispõe sobre o sistema orgânico de produção agropecuária. Diário Oficial da República Federativa do Brasil, Brasília, 23 dez. 2003. Seção 1, pág.8.

CABARET, J.; BOUILHOL, M.; MAGE, C. Managing helminths of ruminants in organic farming. Veterinary Research, v.33, p.625-640, 2002a.

CABARET, J.; MAGE, C.; BOUILHOL, M.. Heminth intensity and diversity in organic meat sheep farms in centre of France. Veterinary Parasitology, v.105, n.1, p.33-47, 2002b.

CARILLO JUNIOR, R. Homeopatia, medicina interna e terapêutica. São Paulo: Santos, 2000. 184p.

CASTILHOS, L.R.; SOUZA, J.C.; PINTO, L.F.; ALBUQUERQUE; FILGUEIRAS, E.P. Avaliação da terapêutica homeopática nas ovariopatias císticas de bovinos leiteiros. Homeopatia Brasileira, v.9, n.1, p.5-15, 2003.

COOP, R.L.; HOLMES, P.H. Nutrition and parasite interactions. International Journal for Parasitology, v.26, p.951-962, 1996.

CCOP, R.L.; KYRIAZAKIS, I. Nutrition-parasite interation. Veterinary Parasitology, v.84, p.187-204, 1999.

DORCHIES, P. Parasite, production et environnement. Bulletin des GTV, v.6, p.21-25, 2000.

HAHNEMANN, S. Doenças crônicas. São Paulo: GEHSP "Benoit Mure", 1835. 202p.

HOSTE, H.; CHARTIER, C. Perspectives de lutte contre les strongyloses gastro-intestinales des ruminants domestiques. Le Point Vetérinaire, v.28, p.181-187, 1997.
HOSTE, H.; DORCHIES, P. Méthode de lutte intégrée contre les parasites em système de production biologique ou conventionnel. Bulletin des GTV, v.8, p.21-24, 2000.

LABRE, P. Homéopathie vétérinaire chez les ovins, bovins et caprins. Villeurbanne: Formation et Edition en Médecines Naturelles Vétérinaires, 2001. 280p.

LARSEN, M. Biological control of helminths. International Journal for Parasitology, v.29, p.139-146, 1999.

OLAVEZ, R.; PIETROSEMOLI, S.; VALERA, Z. Efecto des extracto acuoso de semillas de Neem (Azadirachta indica A. Juss) em el control de coccidiosis (Eimeria sp.) em becerros. In: REÚNION DE LA ASOCIACION LATINO-AMERICANA DE PRODUCCIÓN ANIMAL, 17., 2001, Havana. Anais. Havana: 2001.

PINTO, L.F.; ALMEIDA, B.M. O contexto da homeopatia na pecuária orgânica no Brasil. Homeopatia Brasileira', v.8, n.1, p. 23-28, 2002.

RAGOT, M. Conversion à l'agriculture biologique. Le cas de la production laitière. Dijon: Educagri, 2000. 355p.

REPAB. Réglement Européen des Productions Animales Biologiques. Cahier des Charges concernant le mode de production Biologique des Animaux. Direction des politiues économiques et internationale, Ministère de l'Agriculture et la Pêche, France. 2007.

SÁNCHEZ, M.E.; PERALTA, L.M.; PEDRAZA, V.P. Propuestas de manejo ovino dentro de um sitema de producción orgânica. In: REÚNION DE LA ASOCIACIÓN LATINO-AMERICANA DE PRODUCCIÓN ANIMAL, 17., 2001, Havana, Cuba. Anais. Havana: 2001.

VILLALBA, J.J.; PROVENZA, F.D.; HALL, J.O.; LISONBEE, L.D. Selection of tannins by sheep in response to gastrointestinal nematode infection. Journal of Animal Science, v.88, n.6, p.2189-98, 2010.

WALLER, P.J. Nematode parasite control of livestock in the Tropics/Subtropics: the need for novel approaches. Intertational Journal for Parasitology, v.27, p.1193-1201, 1997.

WALLER, P.J.; THAMSBORG, S.M. Nematode control in green ruminant production systems. Trends in Parasitology, v.20, n.10, p.493-497, 2004,

Recebido em 28/5/11

Aceito em 21/11/12 